\title{
Elastic Body Spline BASEd IMAGE SEGMENTATION
}

Sachin Meena $^{\star} \quad$ V. B. Surya Prasath ${ }^{\star} \quad$ Kannappan Palaniappan ${ }^{\star} \quad$ Guna Seetharaman ${ }^{\dagger}$

${ }^{\star}$ Computational Imaging and VisAnalysis (CiVA) Lab

Department of Computer Science, University of Missouri-Columbia, MO 65211 USA

${ }^{\dagger}$ Air Force Research Laboratory, Rome, NY 13441 USA

\{sm3z5, prasaths, pal\}@missouri.edu, guna@ieee.org

\section{Contribution: Interactive Image Segmentation}

- Elastic body splines (EBS) belonging to the family of 3D splines and were introduced for non-rigid biomedical image registration [1].

- We propose a novel extension of using elastic body splines for learning driven figure-ground segmentation.

- Interactive segmentation with user defined seeds is formulated as learning an interpolating pixel classification function that is then used to assign labels for all unlabeled pixels in the image.

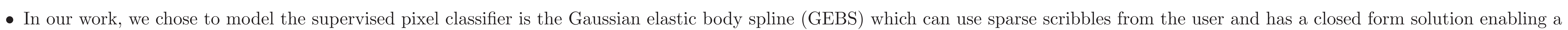
fast on-line implementation.

- The GEBS method for interactive foreground image labeling shows promise and outperforms a previous approach using the thin-plate spline model [2].

\section{Gaussian Elastic Body Splines}

Davis et al [3] used elastic body splines for registration of biomedical images. Given the displacement for landmark points $\vec{p}_{i}$ $i=0, \ldots, N$ between source and target images a transformation is determined that maps the corresponding landmarks from the source to the target image and interpolates the displacement field for all the remaining points in the transformed image. The transformation is given as,

$$
\vec{d}(\vec{x})=\mathbf{A} \vec{x}+\vec{b}+\sum_{i=0}^{N} \mathbf{G}\left(\vec{x}-\overrightarrow{p_{i}}\right) \overrightarrow{c_{i}}
$$

Here $\mathbf{G}$ is the spline function:

$$
\mathbf{G}(\vec{x})=\left[\left((4(1-\nu)-1) \frac{\operatorname{Erf}(\hat{r})}{r}-\sqrt{\frac{2}{\pi}} \sigma \frac{e^{-\hat{r}^{2}}}{r^{2}}+\sigma^{2} \frac{\operatorname{Erf}(\hat{r})}{r^{3}}\right) \mathbf{I}+\left(\frac{\operatorname{Erf}(\hat{r})}{r^{3}}+3 \sqrt{\frac{2}{\pi}} \sigma \frac{e^{-\hat{r}^{2}}}{r^{4}}-3 \sigma^{2} \frac{\operatorname{Erf}(\hat{r})}{r^{5}}\right) \vec{x} \vec{x}^{T}\right] .
$$

Here $\operatorname{Erf}(\cdot)$ is the standard error function, $\hat{r}=|\vec{x}| / \sqrt{2} \sigma$, I is the identity matrix, $\vec{x} \vec{x}^{T}$ is an outer product, $\vec{d}(\vec{x})$ is the displacement vector for the landmark points, $\vec{p}_{i}, \mathbf{A}$ and $\vec{b}$ are the coefficients for the affine transformation and $\vec{c}_{i}$ the set of coefficients for the non-linear elastic terms.

For an interactive image segmentation task we learn the interpolating pixel classification function $\vec{d}(\vec{x})$ in $(1)$. From the user supplied seed points we set

- $\vec{d}(\vec{x})=[+1+1+1]^{T}$ for foreground pixels

- $\vec{d}(\vec{x})=[-1-1-1]^{T}$ for background pixels

- $\vec{x}$ is a feature vector comprised of spatial location and grayscale value of the pixel

Coefficients $\mathbf{A}, \vec{b}$, and $\vec{c}_{i}$ are computed using (1) from the seed points. Let $\mathbf{A} \vec{x}+\vec{b}$, be the affine part of the GEBS interpolating function,

$$
\mathbf{A}=\left[\begin{array}{lll}
\overrightarrow{a_{1}} & \overrightarrow{a_{2}} & \overrightarrow{a_{3}}
\end{array}\right]
$$

Let $\mathbf{W}$ be the vector of all the GEBS coefficients given as,

$$
\mathbf{W}=\left[\begin{array}{llllll}
\mathbf{C}_{F} & \mathbf{C}_{B} & \vec{a}_{1}^{T} & \vec{a}_{2}^{T} & \vec{a}_{3}^{T} & \vec{b}^{T}
\end{array}\right]^{T}
$$

where $\mathbf{C}_{F}$ and $\mathbf{C}_{B}$ are the elastic coefficients corresponding to, $f$, foreground seed pixels and, $b$, background seed pixels,

$$
\mathbf{C}_{F}=\left[\vec{c}_{1}^{T} \ldots \vec{c}_{f}^{T}\right], \quad \mathbf{C}_{B}=\left[\vec{c}_{1}^{T} \ldots \vec{c}_{b}^{T}\right]
$$

The Gaussian Elastic Body Spline (GEBS) mapping determined by the weights $\mathbf{W}$ that we are solving for is given by the relationship

$$
\mathbf{Y}=\mathbf{L} \mathbf{W}
$$

where $\mathbf{Y}$ is the set of displacement vectors for the user defined seeds.

\section{Experimental Results: Segmentation}
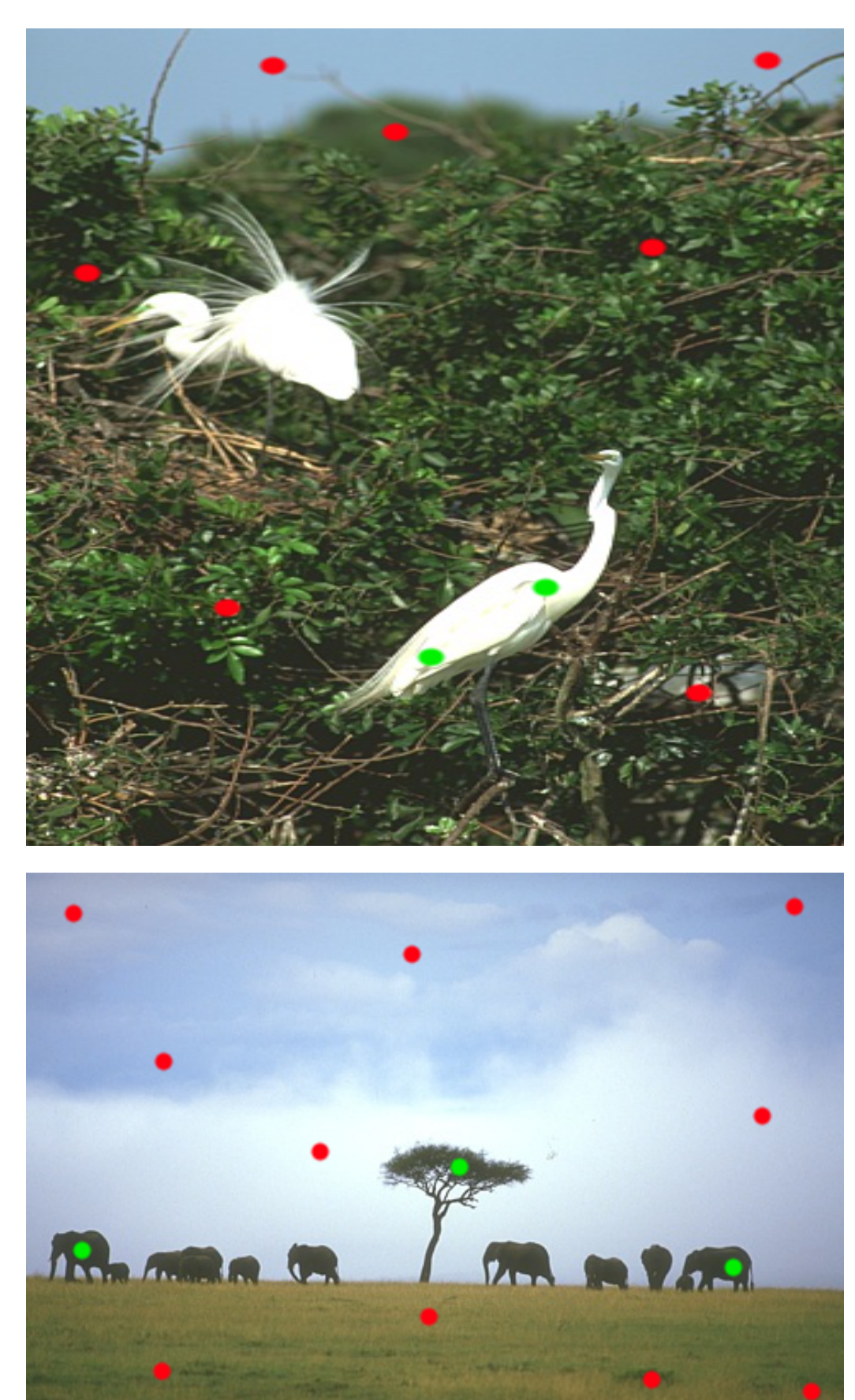

(a) Input with seeds

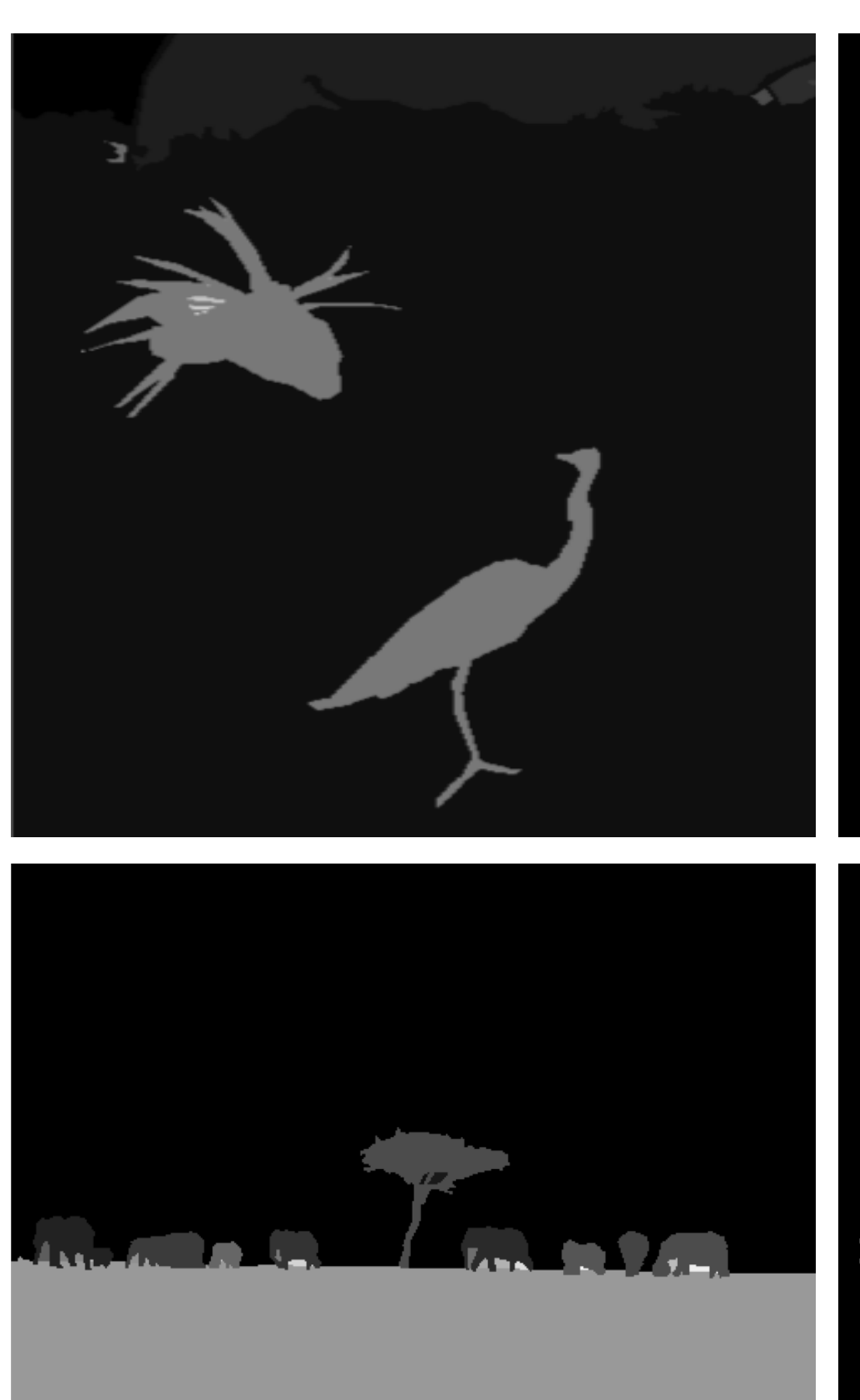

(b) GT

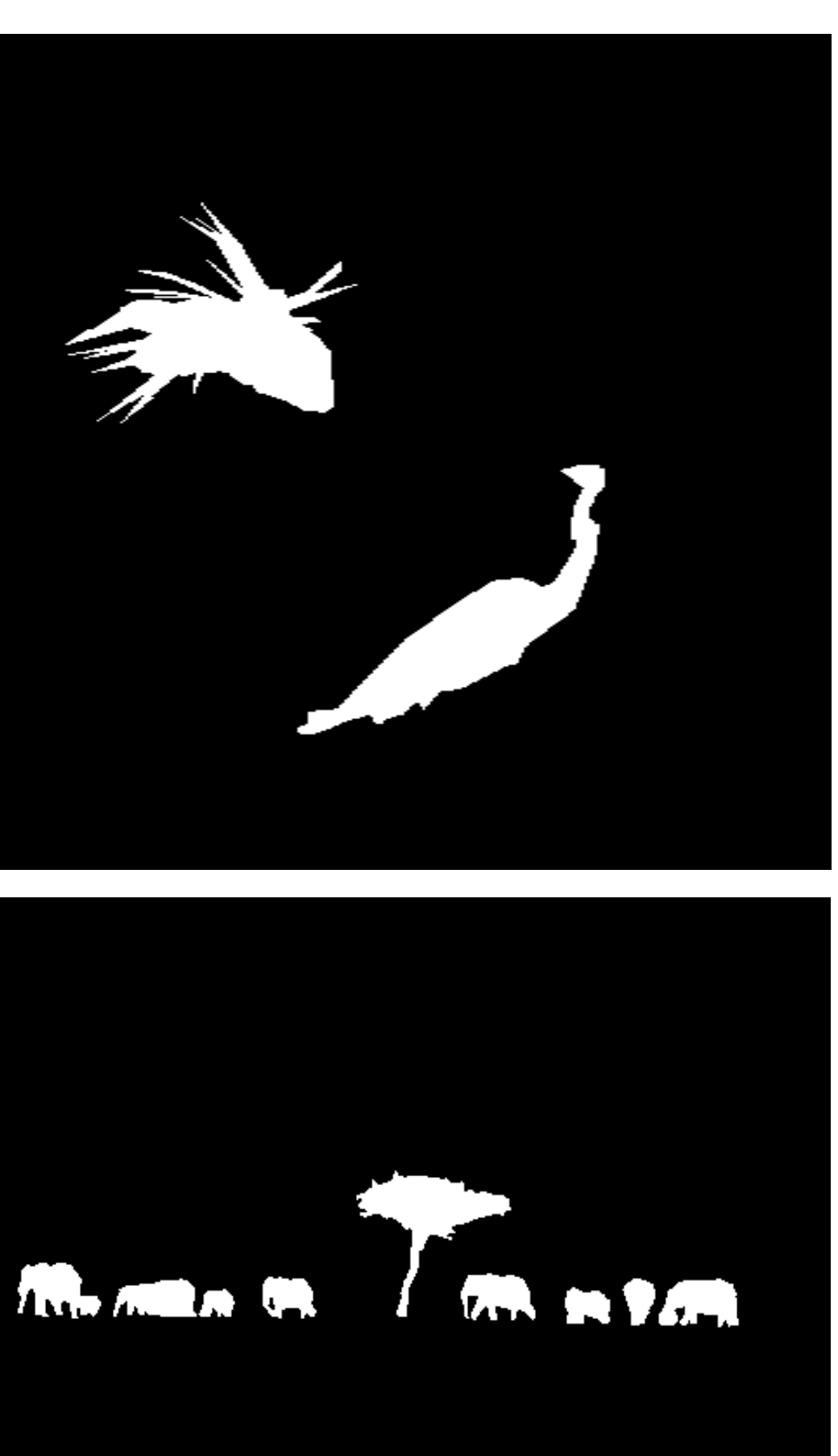

(c) Our GEBS result

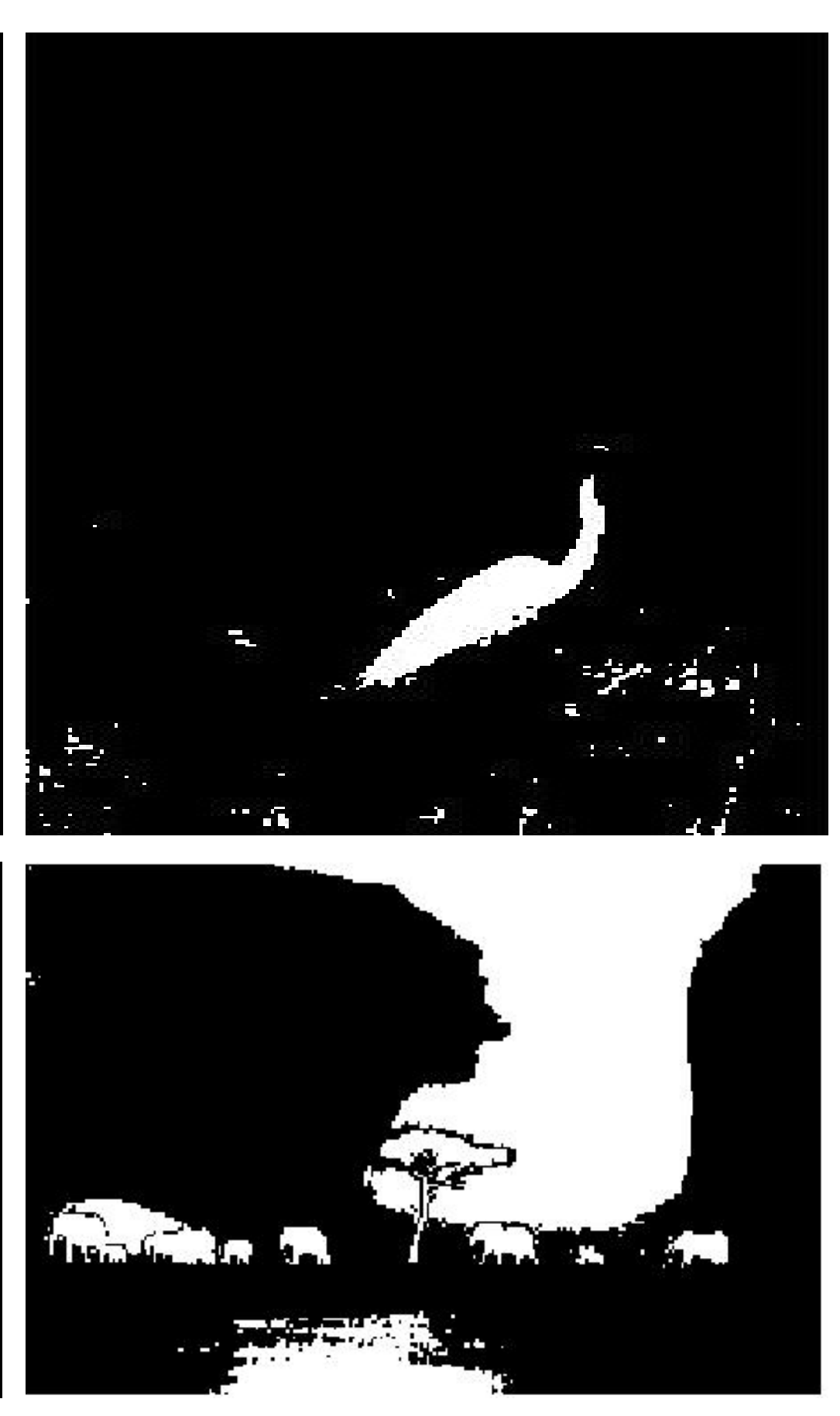

(d) TPS [2] result
Comparison of segmentation results on three BSDS 500 images along with one manual ground-truth. (a) Input image with use provided foreground (green) and background (red) seed points. (b) Sample human annotated ground truth (GT) from BSDS500. (c) Our GEBS segmentation results. (d) TPS segmentation results [2].

\section{REFERENCES}

new class of elastic body splines for nonrigid registration of medical images. Journal of Mathematical Imaging and Vision, vol. 23, no. 3, pp. 253-280, 2005.

2] S. Xiang, F. Nie, C. Zhang, and C. Zhang. Interactive natural image segmentation via spline regression. IEEE Transactions on Image Processing, vol

M. H. Davis, A. Khotanzad, D. P. Flamig, and S. E. Har.
on Medical Imaging, vol. 16, no. 3, pp. 317-328, 1997 .

\section{Interactive Image Segmentation}

The GEBS coefficients are estimated by solving the matrix equation,

$$
\mathbf{W}=\mathbf{L}^{-1} \mathbf{Y}
$$

where,

$$
\begin{gathered}
\mathbf{L}=\left[\begin{array}{cc}
\mathbf{K} & \mathbf{P} \\
\mathbf{P}^{T} & \mathbf{O}
\end{array}\right], \quad \mathbf{K}=\left[\begin{array}{ll}
\mathbf{G}_{F F} & \mathbf{G}_{F B} \\
\mathbf{G}_{B F} & \mathbf{G}_{B B}
\end{array}\right] \\
\mathbf{G}_{F F}(\vec{r})=\left[\begin{array}{ccc}
\mathbf{G}_{11}\left(\vec{r}_{11}\right) & \ldots & \mathbf{G}_{1 f}\left(\vec{r}_{1 f}\right) \\
\vdots & & \vdots \\
\mathbf{G}_{f 1}\left(\vec{r}_{11}\right) & \ldots & \mathbf{G}_{f f}\left(\vec{r}_{f f}\right)
\end{array}\right]
\end{gathered}
$$

with $\vec{r}_{i j}=\vec{x}_{i}-\vec{x}_{j}$ and $\vec{x}_{i}$ is the feature vector for the $i^{\text {th }}$ fore ground seed point. Hence, $\mathbf{G}_{F F}$ is the matrix of GEBS (2) functions defined only over foreground pixels. $\mathbf{G}_{F B}, \mathbf{G}_{B B}$ and $\mathbf{G}_{B F}$ are similarly defined,

$$
\mathbf{P}=\left[\begin{array}{ll}
\mathbf{P}_{F} & \mathbf{I}_{F} \\
\mathbf{P}_{B} & \mathbf{I}_{B}
\end{array}\right]
$$

where the identity matrices are given by,

$$
\mathbf{I}_{F}=\left[\mathbf{I}_{1} \ldots \mathbf{I}_{f}\right], \mathbf{I}_{B}=\left[\mathbf{I}_{1} \ldots \mathbf{I}_{b}\right], \mathbf{I}=\left[\begin{array}{ccc}
1 & 0 & 0 \\
0 & 1 & 0 \\
0 & 0 & 1
\end{array}\right]
$$

for $f$ foreground seed points, $b$ background seed points and,

$$
\mathbf{P}_{F}=\left[\begin{array}{ccc}
x_{11} \mathbf{I} & x_{12} \mathbf{I} & x_{13} \mathbf{I} \\
\vdots & \vdots & \vdots \\
x_{f 1} \mathbf{I} & x_{f 2} \mathbf{I} & x_{f 3} \mathbf{I}
\end{array}\right]
$$

where $x_{i j}$ is the $j^{\text {th }}$ feature for $i^{t h}$ pixel. $\mathbf{P}_{B}$ is similarly defined After solving (5) the GEBS classifier can be estimated and used to interpolate the labels of unlabeled pixels in the image:

$\ell(\vec{x})=\left\{\begin{array}{l}\text { foreground, if majority } \vec{d}(\vec{x}) \text { elements } \geq 0 \\ \text { background, if majority } \vec{d}(\vec{x}) \text { elements }<0 .\end{array}\right.$

\section{Clusters SeParation}
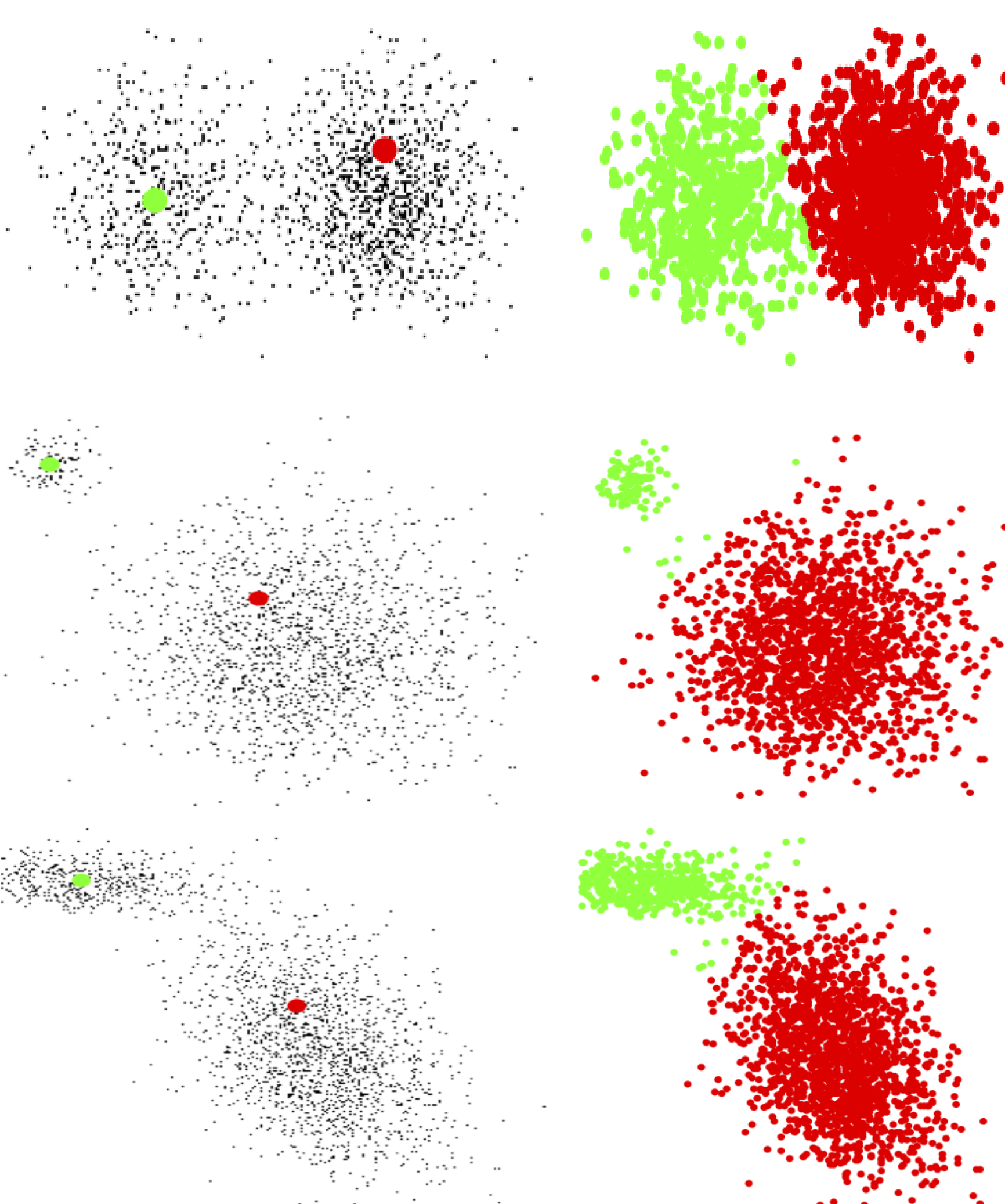

(a) Input with seed

(b) Our GEBS results

Semi-supervised two-class clustering using GEBS. 